\title{
A New Islanding Detection Method for Grid Connected PV System
}

\author{
Ke Jia ${ }^{1}$, Hongsheng $\mathrm{Wei}^{1}$, Yaoqi $\operatorname{lin}^{1}$, Yujin Wang ${ }^{1}$, Guoqing $\mathrm{He}^{2}$ and Bohan $\mathrm{Liu}^{3}$ \\ ${ }^{1}$ North China Electric Power University, Changping Beijing, China \\ ${ }^{2}$ China Electric Power Research Institute State Grid Corporation of China Haidian Beijing, China \\ ${ }^{3}$ State Grid AC Project Construction Co. Ltd, Beijing, China
}

\begin{abstract}
Active detection methods are mostly employed in existing islanding detections for grid connected renewable energy, but there might be non-detection zone in multi-PV system due to mutual interferences of the disturbance signals. In addition, traditional active methods may bring bad influences to power quality of the grid. In view of this, this paper firstly analyzes the failure mechanism of a typical conventional active method based on disturbance in inverters under multi-PV system. Then a novel active islanding detection method suitable for both single photovoltaic (PV) and multiple-PV system is proposed in this paper. It applies an external centralized disturbance algorithm, injecting disturbance signal at PCC and measure the high frequency reactance, which will change abruptly before and after islanding. Moreover, by means of intermittent Zero-CrossingPoint (ZCP) injection control, the method improves accuracy of impedance estimation and reduces disturbances to power system, ensuring good power quality.
\end{abstract}

Keywords-islanding detection; active methods; impedance estimation; zero crossing point

\section{INTRODUCTION}

Renewable energy is rapidly developing around the world; the capacities of photovoltaic and wind turbine are continuing growing in recent years. The connection of renewable energy can ease the energy and environment crisis, however, it also induces some problems to the protection of the grid, and islanding detection is one of them. The DGs and local loads can form an uncontrolled islanding when grid faults or circuit breaker operation occurs (1)-(2). The islanding system may hazard the system equipment and the field operators. Therefore, islanding operating situation should be effectively and quickly detected (3).

According to their working mechanism, islanding detection methods are classified into three categories: the passive methods, the active methods that the communicationbased methods. The passive methods are mainly based on the electrical qualities measured at PCC and their derivative qualities such as voltage amplitude, frequency, rate of change of (ROCO) frequency, harmonic, phase angle, et al. These qualities are easy to obtain, but there exists large nondetection zone (NDZ) and may fail to detect islanding.

The common means of active methods is to inject a disturbance signal to the grid through the inverter, the disturbance signal cause no effect when the PVs are grid connected, but when islanding occurs, the disturbance signal will amplify the change of voltage amplitude, frequency or other qualities and drive them to exceed the threshold, islanding can be detected accordingly. However, in multiinverters system, the disturbance signals may counteract each other, which may lead to the islanding detection failure. Meanwhile, when perturbation occurs in system while the DGs are still grid-connected, the disturbance signals may be amplified and jeopardize the power quality and the system stability.

Communication-based methods mainly consist of remoteend measurement (4)-(6) and wide-area phasor estimation (7)(9). Communication-based methods demand high reliability of real time data transmission, which could cause a large increase of the system investment and might lead to relay mal-function due to failures of communication.

According the drawbacks of conventional islanding detection methods, this paper proposes a centralized high frequency transient injection islanding detection method for both single-PV systems and multi-PV systems. The failure mechanism of the conventional active method is analyzed in detail. The high frequency impedance model of inverter-based $\mathrm{PV}$ is proposed. Disturbance signal is injected at PCC and high frequency impedance is calculates at the same time. The measured impedance will change rapidly before and after islanding, islanding detection is on this basis. By means of Zero Point Crossing (ZPC) injection, islanding detection can be detected accurately and quickly.

\section{Failure Mechanism of CONVENTIONAL ACtiVE METHOd BASED ON INVERTER DisTURBANCE}

\section{A. Frequency-Reactive Power Feedback METHOD}

After islanding, the voltage amplitude at PCC relates to active power and the frequency relates to reactive power. The frequency-reactive power ( $\mathrm{f}-\mathrm{Q}$ ) feedback method is one of the conventional inverter-based active islanding detection (10)(12). F-Q feedback method modifies the control strategy of PLL and output certain reactive power to create a large change of frequency. Islanding can be detected by the change of frequency at PCC caused by reactive power disturbance outputted by the inverter. The outputted reactive power is based on the difference of PCC voltage frequency and grid rated frequency. In order to obtain sufficient reactive power 
disturbances from a small change in frequency, which is to improve the detection speed, a frequency feedback is added in reactive power disturbance. This will enlarge the frequency differences even if the local loads' consuming power matches with the PVs' output power. A typical grid connected inverterbased PV system with local paralleled loads is simplified and shown in the Figure I. According to the amplitude and frequency variations of inverter's output voltage, the islanding situation can be detected. The inverter, as shown in the Figure I, is controlled for maximum real power output while the reactive power is set to be zero for normal operation.

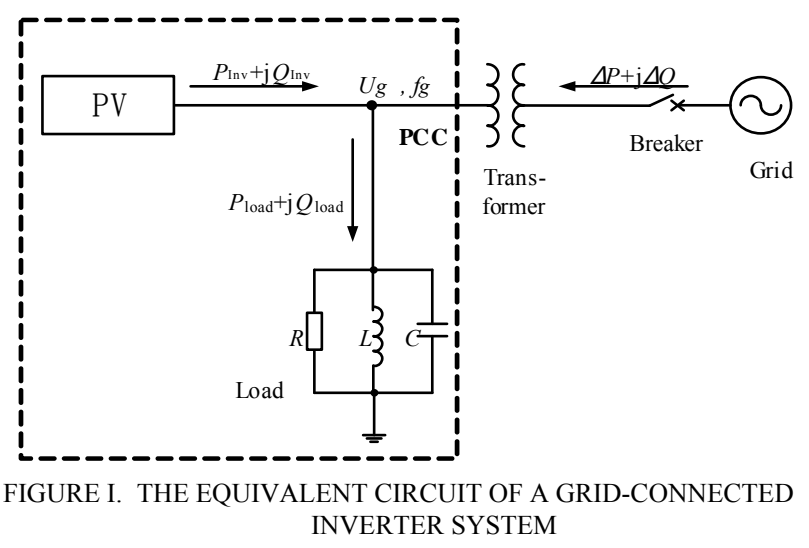

In Figure I, the inverter will be connected to the grid when breaker is closed. At this time, there will be:

$$
\begin{gathered}
P_{\text {load }}=\frac{3 U_{g}^{2}}{R}=P_{i n v}+\Delta P \\
Q_{\text {load }}=3 U_{g}^{2}\left(\frac{1}{2 \pi f_{g} L}-2 \pi f_{g} C\right)=Q_{i n v}+\Delta Q
\end{gathered}
$$

Whereby, Pload and Qload are the active and reactive power consumed by loads in normal operation; Pinv and Qinv are the inverter's active and reactive power outputs; $\Delta \mathrm{P}$ and $\Delta \mathrm{Q}$ are the active and reactive power supplied by the grid; $\mathrm{Ug}$ and fg are the amplitude and frequency of the inverter's output voltage.

The local loads will be supplied by the PV system when breaker is open and islanding occurs. Uisland and fisland denote the amplitude and frequency of the terminal voltage of the local loads, P'load and Q'load are the active and reactive power consumed by the local loads. When the inverter operates on constant power control mode while islanding, there will be:

$$
P_{\text {load }}^{\prime}=\frac{3 U_{\text {island }}^{2}}{R}=P_{\text {inv }}
$$

$$
Q_{\text {load }}^{\prime}=3 U_{\text {island }}^{2}\left(\frac{1}{2 \pi f_{\text {island }} L}-2 \pi f_{\text {island }} C\right)=Q_{\text {inv }}
$$

The terminal voltage of local load in islanding operation can be deduced by formula (5):

$$
U_{\text {island }}=\sqrt{\frac{P_{i n v} R}{3}}
$$

When the inverter works in unity power factor mode and $Q_{i n v}=0$, the inverter won't supply reactive power to the loads after islanding. Thus, the frequency at PCC can be represented by (6):

$$
f_{\text {island }}=\frac{1}{2 \pi \sqrt{L C}}
$$

Therefore, the relationship between $\Delta Q$ (the mismatching reactive power) and $f_{\text {islanding }}$ can be expressed as:

$$
\frac{\Delta Q}{P_{\text {inv }}}=\left(\frac{U_{g}}{U_{\text {island }}}\right)^{2} Q_{f}\left(\frac{f_{\text {island }}}{f_{g}}-\frac{f_{g}}{f_{\text {island }}}\right)
$$

where $Q_{f}=R \sqrt{\frac{C}{L}}$ is the quality factor of the load

As shown in (7), the reactive power difference between inverter's output and the local load will result in frequency variation during islanding.

From the analysis above, the frequency is related to the reactive power output of the inverter after islanding. Therefore, the grid-connected inverter system's islanding state can be detected by frequency change utilizing reactive power disturbance outputted by inverter. The control of reactive power disturbance is based on the difference of PCC voltage's frequency and grid voltage's rated frequency. In order to obtain sufficient reactive power disturbances from a small change in frequency, which is to improve the detection speed, a frequency feedback is added in control of reactive power disturbance, named the three-phase frequency-reactive power feedback method. The feedback is set as formula (8) and the control block diagram is shown in Figure II.

$$
Q_{i n v}=k\left(f_{a}-f_{g}\right)
$$

Where $k$ is the frequency feedback coefficient, $f_{a}$ is the frequency of the voltage at the point of common coupling (PCC), $f_{g}$ is the rated frequency of the main grid $(50 \mathrm{~Hz})$. 


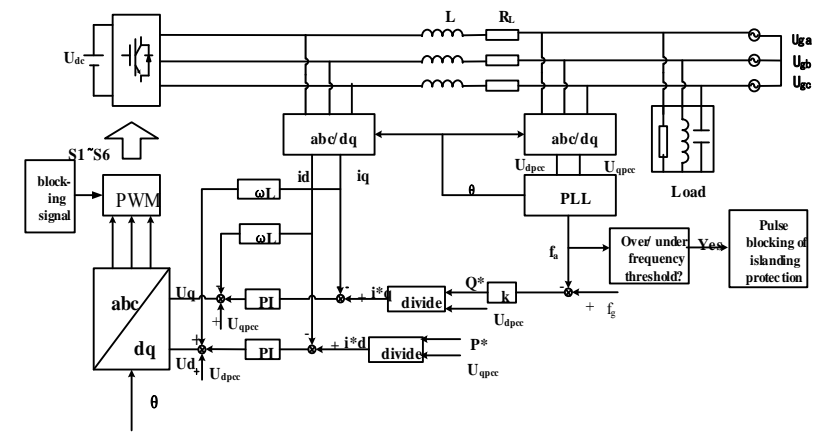

FIGURE II. CONTROL STRATEGY OF F-Q FEEDBACK METHOD IN THE INVERTER

As shown in the Figure II, by using constant power control, the active and reactive power output of the PV inverter are constants. Voltage at PCC of the PV system, clamped by the main grid, is the rated voltage and the corresponding values $U_{d p c c}, U_{q p c c}$ in d-q coordinate system. Accordingly, reference current $i_{q}^{*}$ and $i_{d}^{*}$ can be calculated from the outer power control loops. The current state feedback values, $\omega L i_{d}$ and $\omega L i_{q}$, are introduced to achieve decoupling. The inner current loop outputs the reference voltages $U_{d}$ and $U_{q}$ which generate Pulse-Width Modulation (PWM) signals after the dp/abc conversion.

The PCC voltage's frequency changes very little after islanding if local load matches power output of the inverter to a high degree. By introducing in the feedback of frequencyreactive power, reactive power disturbance of load, reactive power offset of filter capacitor and linked reactor in the inverter will be magnified due to the feedback's effect, making the frequency exceed the allowed range and islanding protection take action.

\section{B. Validity and Failure Mechanism of $f-Q$ Feedback Method}

To study on the performances of the f-Q feedback method in the islanding detection, the simulation model is built in Matlab/Simulink according to the system structure introduced in the Figure I and control strategy presented in the Figure II and formula (8). Detailed simulation parameters are: the inverter's output is stepped up via transformers to a $220 \mathrm{kV}$ system of infinite capacity. The active power output of the inverter is $300 \mathrm{~kW}$ and the reactive power output is determined by the frequency difference, that is $Q_{i n v}=k\left(f_{a}-50\right)$ Var, where the feedback factor is $k=100^{2}$. Make the power outputted by PV and consumed by load match, and itis the NDZ of passive methods. All the islanding simulations investigated in this paper are in the "power matching" condition, this is to prove the advance of active method when passive methods fail to detect islanding. Islanding is set at $0.2 \mathrm{~s}$ and the simulation duration is $1.2 \mathrm{~s}$. Without the $\mathrm{f}-\mathrm{Q}$ feedback method, the frequency will remain around $50 \mathrm{~Hz}$ after islanding because of the highly matching between the inverter output power and the power consumed by the local loads.

Apply three-phase f-Q feedback method to detect islanding at power matching situation, the results are shown the in Figure III.

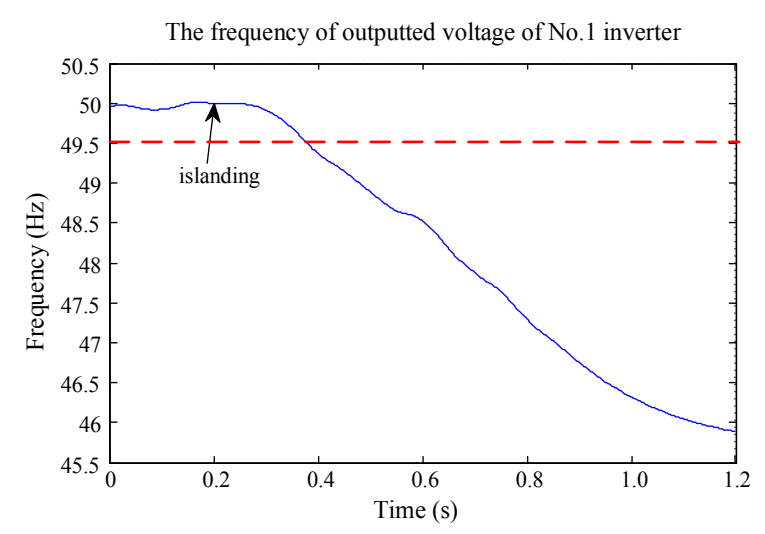

a) Frequency of voltage at the outlet of the inverter

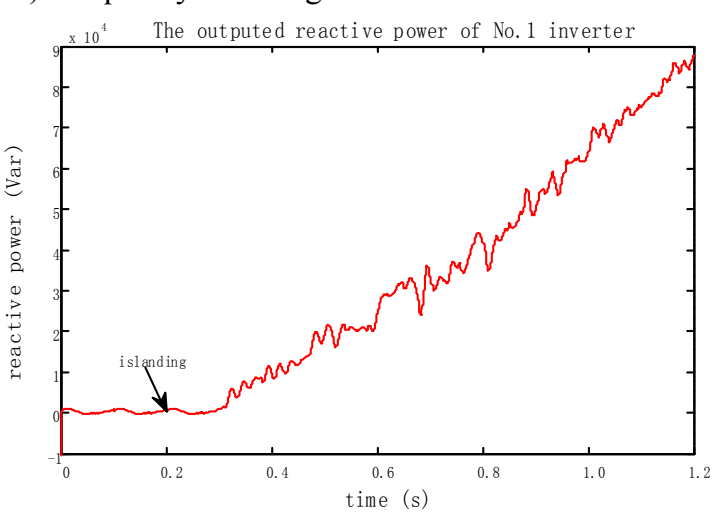

b) Outputted reactive power of the inverter

FIGURE III. FREQUENCY MEASURED AT THE OUTLET OF THE INVERTER

As shown in Figure III, due to the f-Q feedback algorithm, little change of frequency can result in large change of reactive power output of inverter. As the frequency drops, the outputted reactive power increases, which will accelerate the further drop of frequency. The frequency of the system drops to less than $49.5 \mathrm{~Hz}$ within $0.2 \mathrm{~s}$ after islanding, and the islanding operation state of the system can be detected in short time. Under certain circumstance (feedback factor is beyond certain value), f-Q feedback method can have good accuracy and speed to detect islanding.

However, in order to cooperate with low voltage run through (LVRT), the feedback factor of f-Q method is set to be small (if the f-Q method is too sensitive, LVRT may fail and large range of inverter will be off-line). This will result in a relatively small output of reactive power after islanding, and the frequency change is not significant enough to detect islanding situation, active islanding may fail at this situation. Setting feedback factor $k=20^{2}$, islanding occurs at $1 \mathrm{~s}$ and simulation duration is $1.5 \mathrm{~s}$, the result is shown as following in Figure IV. 


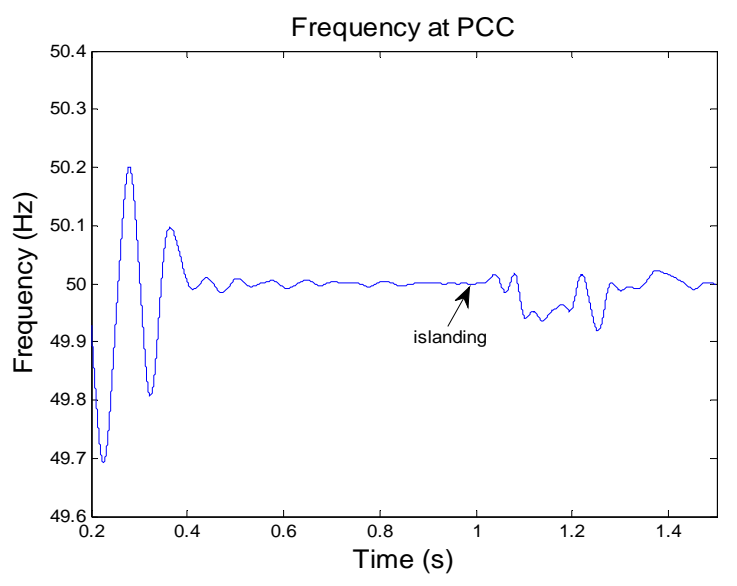

a) The frequency of voltage at the outlet of 1 inverter

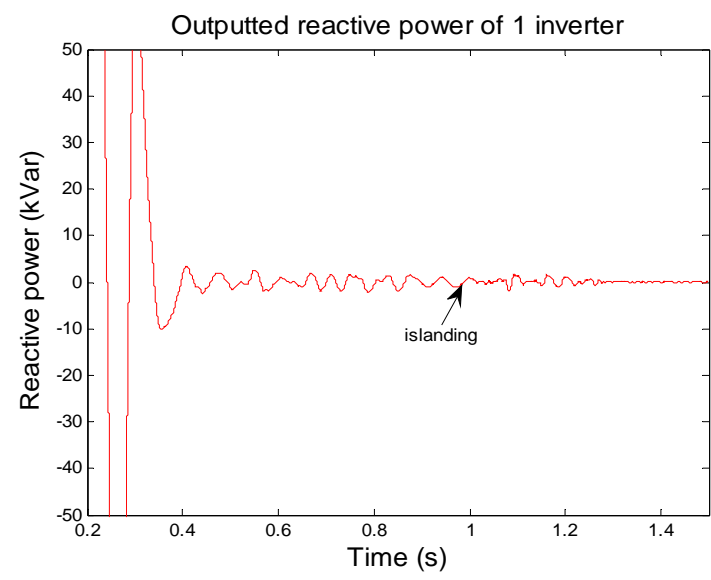

b) The output reactive power of 1 inverter

FIGURE IV. THE FREQUENCY AND REACTIVE POWER CURVES IN MULTI-INVERTER SYSTEM DERIVED USING THE F-Q FEEDBACK METHOD

As shown in the Figure IV, due to the small feedback factor, the outputted reactive power inverters fluctuates in a small scale and the summation of the output feedback reactive power from all inverters is close to zero. The frequency curve fluctuates within a narrow range around $50 \mathrm{~Hz}$ and does not exceed the islanding detection threshold. The change of frequency is not significant enough to detect islanding, and f$\mathrm{Q}$ feedback method will fail to detect islanding.

The drawbacks of the conventional active islanding detection methods are as follows: 1) if the feedback factor is set to be small, the outputted reactive power is too small to induce enough change of frequency, and islanding detection may fail. 2) If the feedback factor is set too big, when the fluctuation occurs in the system while PV system is grid connected, the summation of all the feedback reactive/active power may be large enough to induce the instantaneous change of frequency/voltage, and power quality issues will occur. 3) The conventional active islanding detection methods output certain reactive power due to the difference between frequency at PCC and rated frequency. It ignores the reactive loss while they flow through the transmission lines and transformers, considering the reactive power loss, the reactive power transmitted to the PCC may be opposite to the power outputted by the inverters, this will leads to the failure of islanding detection.

\section{ISLANDING DETECTION FOR MULTI-PV SYSTEM}

\section{A. Rationale of Impedance Estimation Method}

Equivalent schematic of impedance estimation method using external centralized disturbances is shown as Figure V. It applies independent centralized disturbance injection at PCC, other than conventional disturbance injection methods based on injection through inverters.

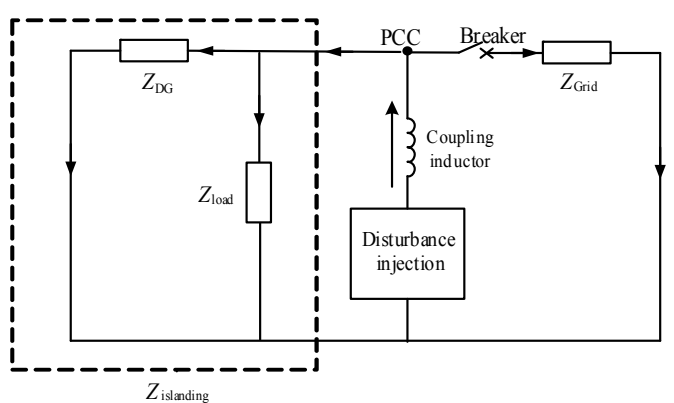

FIGURE V. SCHEMATIC OF EQUIVALENT SYSTEM IMPEDANCE UNDER EXTERNAL CENTRALIZED DISTURBANCES

Its measured impedance characters at injection point and basic principle of detection are stated as follows:

Usually, the equivalent impedance of grid is much smaller than that of PV and load in a grid-connected distributed generation system because of their capacity differences.

$$
\begin{gathered}
Z_{\text {Grid }}<<Z_{D G}, Z_{\text {Grid }}<<Z_{\text {load }} \\
Z_{\text {Islanding }}=\frac{Z_{D G} Z_{\text {load }}}{Z_{D G}+Z_{\text {load }}}>>Z_{\text {Grid }}
\end{gathered}
$$

Where $Z_{\text {Grid }}, Z_{P V}$ and $Z_{\text {load }}$ are the equivalent grid impedance, $\mathrm{PV}$ impedance and the load impedance in the high frequency. $Z_{\text {islanding }}$ is the measured islanding impedance in the high frequency. Before islanding, the measured impedance $Z_{\text {meas }}$ at PCC is:

$$
Z_{\text {meas }}=\frac{Z_{\text {Grid }} Z_{\text {Islanding }}}{Z_{\text {Grid }}+Z_{\text {Islanding }}}<Z_{\text {Grid }}
$$

After islanding, the measured impedance $Z_{\text {meas }}^{\prime}$ at PCC is:

$$
Z_{\text {meas }}^{\prime}=Z_{\text {Islanding }}>>Z_{\text {Grid }}>Z_{\text {meas }}
$$


Equations (11)-(12) indicate that the measured impedance shows PV impedance, load impedance and the grid impedance in parallel during normally operation and this value after islanding (load impedance and the PV impedance in parallel) will be larger than normal operation. In this method, there is only one disturbance source in the whole system, avoiding mutual interferences of disturbances generated by multiple inverters. Thus, it is suitable for multi-inverter distributed generation system. Moreover, due to employing independent disturbance injection, the method is also suitable for power supply directly connected to grid like synchronous generator.

\section{B. Injection Control and Reactance Calculation}

As mentioned in the section above, external centralized disturbance injection is proposed for islanding detection in this paper. The disturbance generating and injecting device is implemented by utilizing the single-phase full-bridge inverter circuit, shown in Figure VI.

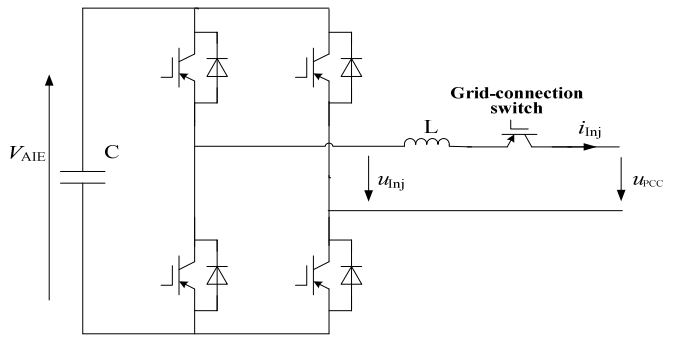

FIGURE VI. SCHEMATIC OF INDEPENDENT DISTURBANCE GENERATING DEVICE

As shown in Figure VI, a capacitor $C$ of constant DC voltage is connected with bridges of single-phase full-bridge inverter. The inverter is connected to phase A and B of the system at PCC, via a considerable coupling inductor L whose inductance value is set according to the magnitude of injection current. By switching control of IGBT, the inverter produces square pulse voltage, forms a triangular current 'spike' through the coupling inductor L, shown in Figure VII. Width of the injection current spike is controllable, so it can realize intermittent injection, which can greatly reduce the disturbance injected into the grid, compared with persistent injection of specific-frequency current. In addition, the current signal contains abundant and uninterrupted frequency components, provide basis for the wide-band high-frequency reactance calculation.

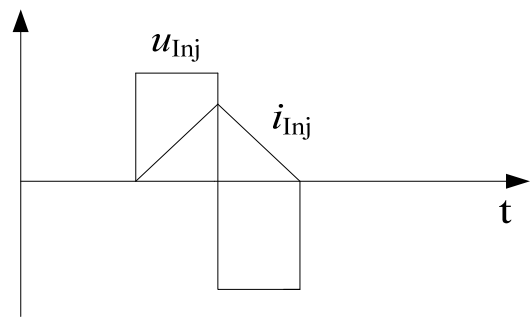

FIGURE VII. CURVE OF VOLTAGE AND CURRENT DISTURBANCE GENERATED BY DISTURBANCE GENERATING DEVICE
In terms of injection strategy, this paper proposes ZeroCrossing-Point (ZCP) injection control method. It detects ZCPs of the voltage at PCC and injects a current spike mentioned above $\left(i_{\text {Inj }}\right)$ into the system at each ZCP or some ZCPs, measuring the PCC voltage $u_{\mathrm{PCC}}$ in the meantime for impedance calculation. $u_{\mathrm{PCC}}$ is a superposed current composed of inherent system voltage which is viewed as noise component for impedance calculation and voltage response to injected current $i_{\text {Inj }}$ which is indeed the effective voltage component for impedance calculation. Because the pulse is injected at ZCPs, the voltage response to $i_{\text {Inj }}$ (the effective voltage component) is the major component, and the inherent system voltage component of $u_{\mathrm{PCC}}$ is minimized to nearly zero and can be ignored. That will improve signal to noise ratio (SNR) and the precision of impedance estimation, as well as lower the injected disturbance current magnitude required for effective calculation.

In addition, the DC voltage $V_{\mathrm{AIE}}$ (shown in Figure VI) of the disturbance injection device connected to the system is required to be greater than system voltage at PCC, otherwise the anti-paralleled diodes in the injection device will be turned on by high system voltage. This brings about a strict requirement for DC voltage level and the capacitor of DC part as well as the insulation. Given this, the paper utilizes a collaboration of ZCP injection control and grid-connection control to solve the problem. When detecting the system voltage approaching zero, the grid-connection switch (a fullcontrolled IGBT, shown in Figure VI) is controlled to be connecting-state before the ZCP injection starts and then be disconnected after disturbance injection is finished. As a result, $V_{\text {AIE }}$ just need to be higher than maximum value of voltage limited within the short injection duration, which is around zero value. This will largely increase the practicability and economic efficiency.

\section{Simulation Verification Results}

Matlab simulation software is employed in research of this paper. System model as Figure V is built in simulation to verify the islanding detection method and model proposed by this paper. The PV capacity is $1 \mathrm{MW}$ and the RLC load capacity is $1 \mathrm{MW}$ as well (to make the PV power match with the load demand and this leads to failure of the passive method). Power source of the grid side is an ideal voltage source connected to $220 \mathrm{kV}$ transmission line and then a $35 \mathrm{kV}$ distribution line. The capacity of the transformer on the grid side is 50MVA and the capacity of the transformer for PV is 5MVA.

\section{A. Simulation Result of Single-PV Condition}

The impedance characteristic in time domain is shown in Figure VIII, Results of the wide-band calculation are reduced to reactance value at $4 \mathrm{kHz}$, the calculated impedance changes more obviously at high frequency range which is more easily to distinguish islanding from grid connected condition. . 


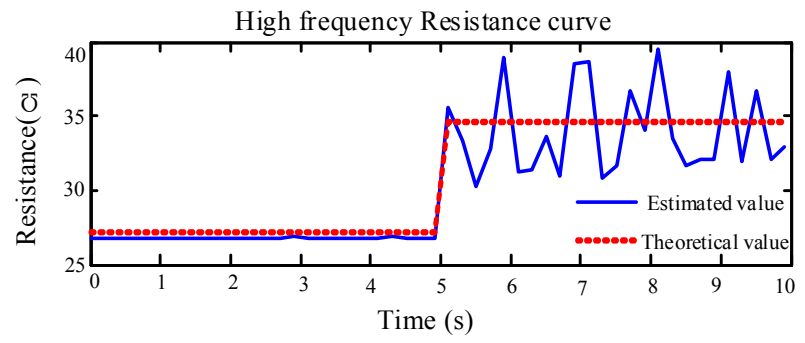

a) Change of resistance before and after islanding

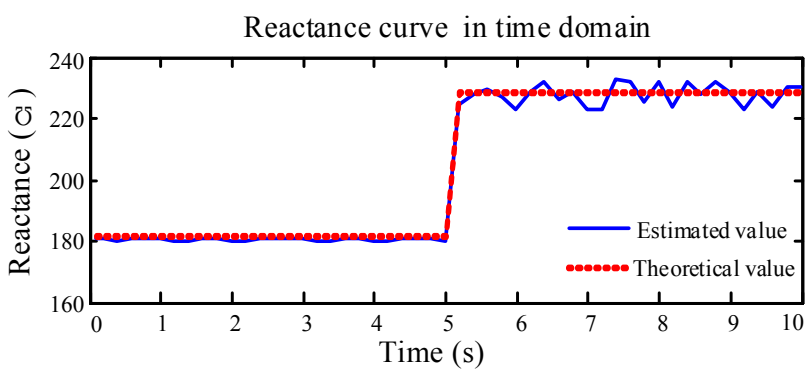

b) Change of reactance with ZCP injection

\section{FIGURE VIII. ON-LINE IMPEDANCE CURVE IN TIME DOMAIN}

As shown in the Figure VIII, islanding occurs at $5 \mathrm{~s}$. Subgraph a) is $R$ characteristic curve by on-line impedance calculation, and it changes after islanding but with large calculation errors due to a poor frequency response in the high frequency domain. The Subgraph b) is $L$ characteristic curve by on-line impedance calculation, demonstrates the reactance derived by the proposed ZCP injection, step change occurs within $0.2 \mathrm{~s}$ and islanding status is detected quickly and effectively.

Comparing with the results of high frequency resistance, the reactance estimation with ZCP injection gives more accuracy due to less system noise is involved in each of the measuring windows. This would lead to improvement in the sensitivity of the islanding detection. The proposed injection method takes place at ZCPs and uses the average value of several injection results for islanding detection. This can enhance the accuracy and avoid the side effect of noise and calculation error.

\section{B. Simulation Result of Multi-PV Condition}

The proposed islanding detection method is also suitable for multi-PV (three PVs) situation where the $\mathrm{f}-\mathrm{Q}$ active islanding detective method fails to work as presented in the Section II. The total outputted power of the three PVs is $3 \mathrm{MW}$, the active power consumed by local loads is $3 \mathrm{MW}$ as well, the reactive power consumed by local loads is fully compensated by paralleled capacitors. Figure IX a) and b) show the estimated reactance result of the system with $3 \mathrm{PVs}$ and $1 \mathrm{PV}$ applying different control strategies (different $\mathrm{Kp}$ and $\mathrm{Ki}$ parameters) and different output powers. As analyzed in section A, high frequency reactance is more accuracy to detect islanding, choose high frequency reactance is enough to distinguish islanding of multi-PV system.
High frequency Reactance curve

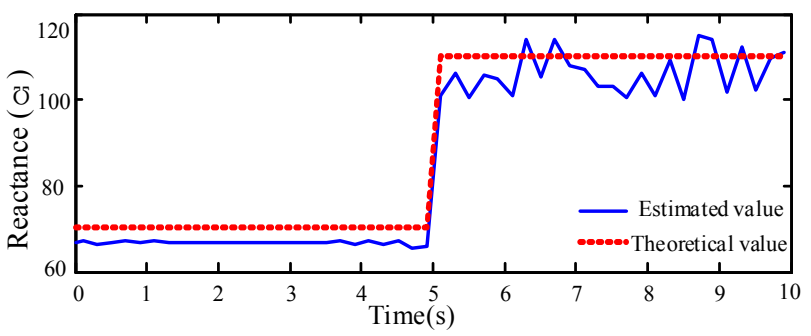

FIGURE IX. ON-LINE REACTANCE CHARACTERISTIC IN THE CASE OF 3PVS

As shown in Figure IX, islanding occurs in the grid connected multi-PV (three-paralleled PV) system at $5 \mathrm{~s}$, the measured high frequency reactance changes abruptly within $0.2 \mathrm{~s}$ while applying the islanding detection method proposed in this paper. Islanding status can be detected quickly and effectively through its abrupt change. In addition, the estimated results match very well with theoretical values, and the proposed method is proved by the theoretical result and simulation result as well. This is attributed to ZCP injection and high frequency impedance estimation, islanding detection method is efficient and accuracy.

\section{CONCLUSIONS}

As the grid connected PVs system develops, conventional islanding detection methods mainly which aim at detecting islanding of single-PV system are facing challenges. This paper proposed an islanding detection method based on high frequency impedance estimation using external centralized transient injections. It avoids detection failure caused by mutual interferences of multi-supply disturbance signals and it is universally applicable for different types of grid-connected inverters and synchronous generators with no inverters. The simulation results show good accuracy and efficiency of the proposed islanding detection methods.

By applying ZCP injection control and wide-band highfrequency reactance calculation, the method amplifies the change of the reactance before and after islanding, and it will provide good islanding detection accuracy. Moreover, the intermittent ZCP injection control of short tiny current spikes, proposed in the paper, greatly reduces the disturbance volume injected into power system caused by active detection method, ensuring good power quality.

\section{ACKNOWLEDGMENT}

This work was supported in part by the National program on key basic research project (973 Program, No. 2012CB215206), by the NSFC project under Grant 51407067, the "111" Project under Grant B08013 and FRFCU 2016ZZD01, by the Science and technology project "Research and application on centralized anti-islanding technology of large scale renewable energy". 


\section{REFERENCES}

[1] Adrian V. Timbus, Remus Teodorescu and Frede Blaabjeig. "Online Grid Impedance Measurement Suitable for Multiple PV Inverters Running in Parallel". IEEE Applied Power Electronics Conference and Exposition, 2006.

[2] H. G. Far, A. J. Rodolakis, and G. Joos, "Synchronous distributed generation islanding protection using intelligent relays," IEEE Trans. Smart Grid, vol. 3, no.4, pp.1695-1703, Dec. 2012.

[3] ChengQi-ming, WangYing-fei, ChengYin-man, etal."Overviewstudy on islanding detectingmethods for distributed generation grid-connected system”.Power System Protection and Control. vol.6, pp.147-154, 2011.

[4] YU B, MATSUI M, and SO J.”A high power quality anti-islanding method using effective power variation". Solar Energy, 82(4): 368-378, 2008.

[5] Chun Li, J. Savulak, and R. Reinmuller, "Unintentional islanding of distributed generation-operating experiences from naturally occurred events," IEEE Trans. Power Delivery, vol. 29, no.1, pp. 269-274, Feb. 2014.

[6] Valentini, M., Munk-Nielsen, S.and Valderrey Sanchez, F. "A new passive islanding detection method for grid-connected PV inverters," Power Electronics, Electrical Drives, Automation and Motion, 2008 SPEEDAM 2008. International Symposium on , vol., no., pp.223, 228, 2008

[7] W. R. Issa, M. A. Abusara, and S. M. Sharkh, "Control of transient power during unintentional islanding of microgrids," IEEE Trans. Power Electronics, vol. 30, no.8, pp. 4573-4584, Aug. 2015.

[8] Yafaoui, A., Bin Wu, Kouro, S., "Improved Active Frequency Drift Anti-islanding Detection Method for Grid Connected Photovoltaic Systems," Power Electronics, IEEE Transactions on , vol.27, no.5, pp.2367,2375, May 2012.

[9] H. Laaksonen, “Advanced islanding detection functionality for future electricity distribution networks," IEEE Trans. Power Delivery, vol. 28, no. 4, pp. 2056-2064, Oct. 2013.

[10] Laaksonen, H., "Advanced Islanding Detection Functionality for Future Electricity Distribution Networks," Power Delivery, IEEE Transactions on, vol.28, no.4, pp.2056,2064, Oct. 2013

[11] M. R. Alam, K. M. Muttaqi, and A. Bouzerdoum, "A multifeature-based approach for islanding detection of DG in the subcritical region of vector surge relays," IEEE Trans. Power Delivery, vol. 29, no. 5, pp. 2349-2358, Oct. 2014.

[12] B. Matic-Cuka, and M. Kezunovic, "Islanding detection for inverterbased distributed generation using support vector machine method," IEEE Trans. Smart Grid, vol. 5, no. 6, pp. 2676-2686, Nov. 2014.

[13] Zeineldin H H, Kennedy S. "Sandia frequency-shift parameter selection to eliminate nondetection zones". IEEE Transactions Power Delivery, 24(1): 486-487, 2009.

[14] Hesari, S.A.S.; Hamzeh, M.; Toobak, H., "Performance assessment of an impedance based islanding detection method in a distribution network with multiple PV inverters,"Power and Energy Systems (ICPS), 2011 International Conference on, vol., no., pp.1,6, 22-24 Dec. 2011. 